\title{
The relation between fibrosis of hilar lymph glands and the development of parenchymal silicosis
}

\author{
J Murray, I Webster, G Reid, D Kielkowski
}

\begin{abstract}
The necropsy findings on the cardiorespiratory organs of 849 South African gold miners were analysed to test the hypothesis that fibrosis of the hilar lymph glands predisposes to the development of parenchymal silicotic nodules. Four hundred and eighty three cases had fibrosed glands, $34 \%$ of which also had parenchymal silicosis. By comparison, of 238 cases with silicosis, $88 \%$ had fibrosed glands. The proportion of cases with both silicosis and fibrosed glands, as well as those with gland fibrosis only, increased with increasing duration of exposure. As far as can be ascertained from a necropsy series such as this, it is possible that fibrosis of the lymph glands may predispose to the development of lung parenchymal silicosis.
\end{abstract}

The fate of inhaled particles (including quartz) deposited in the lower respiratory tract has been widely studied. ${ }^{1-4}$ The question of whether or not fibrosis of the hilar lymph glands is associated with the development of lung parenchymal silicosis, however, has not been specifically considered. Most particles that reach the alveoli are rapidly cleared by mucociliary transport, either in macrophages or as particulate matter. Particles that are not removed by the mucociliary escalator pass from the alveolar space into the interstitial tissue and from there into the perivascular lymphatic channels. They are then translocated to the regional lymph glands either as free particles or in macrophages. . $^{5-8}$

Dust that is not removed either via the mucociliary escalator or by lymphatic clearance is retained in the lung. Here, interaction occurs between quartz particles and pulmonary macrophages resulting in a cascade of inflammatory and fibrotic events with the eventual formation of silicotic nodules. ${ }^{-11}$

National Centre for Occupational Health, PO Box 32492, Braamfontein, 2017, Republic of South Africa J Murray, I Webster, G Reid, D Kielkowski

\section{Hypothesis}

It is suggested that fibrosis of the hilar nodes, whether due to silicotic collagenisation or other causes, will cause obstruction to the lymphatic drainage of the lung. This will decrease clearance, increase accumulated lung dust burden, and hence predispose to the development of parenchymal silicosis.

\section{Methods}

\section{STUDY POPULATION}

Necropsy examination of the cardiorespiratory organs of all miners and ex-miners who die in South Africa is required by law provided the next of kin consents. Current estimates indicate that $86 \%$ of white goldminers who die in service or in retirement undergo necropsy. Necropsy rates for black miners and ex-miners are unknown but are probably much lower that for white miners. For this reason, the study was limited to white miners.

Data were extracted from necropsy reports of all miners whose service was limited to goldmines, who had no pneumoconiosis other than silicosis, and whose lungs were examined during 1988.

\section{INFORMATION ON EXPOSURE}

Each miner's exposure was categorised from his job record. This information was derived from service records from the approximately 80 goldmines at which the man might have worked and is supplied by the Medical Bureau for Occupational Diseases to complete the file record of all miners and ex-miners who die. These exposure data and the necropsy results are recorded in a computerised database PATHAUT. ${ }^{12}$

\section{INFORMATION ON PATHOLOGY}

Data were extracted from the computerised necropsy report of each case. Necropsy examination that is performed by pathologists at the National Centre for Occupational Health routinely includes histological assessment of tissue from a main bronchus, hilar lymph glands, and upper, mid, and lower zones of both lungs. Using these pathological data, each miner was categorised according to the presence or absence of fibrosed hilar glands and parenchymal silicosis.

Silicosis was defined as the presence of nodules of whorled concentric fibrous connective tissue in the 
lung parenchyma. Fibrosed hilar glands included the fibrosis of silicotic collagenisation, that of healed tuberculous lymphadenitis, and non-specific fibrosis resulting from other causes.

ANALYSIS

Statistical Analysis Software, Personal Computers (PCSAS) was used. Logistic regression was performed and the CATmode procedure was used. ${ }^{13}$ The odds ratios and $95 \%$ confidence intervals $(95 \%$ CI) were calculated using the formulae: odds ratio $=$ antilog $(\beta)$ coefficient; $95 \% \mathrm{CI}$ : lower limit = antilog $\left(\beta-\mathrm{SE}_{\beta} \times 1.96\right)$, upper limit $=$ antilog $\left(\beta+\mathrm{SE}_{\beta} \times\right.$ $1 \cdot 96)$.

\section{Results}

The study population consisted of 849 cases.

The cases were analysed in a two by two table according to the presence or absence of parenchymal silicosis and hilar gland fibrosis (table 1). Of the 849 cases, $483(56.9 \%)$ had fibrosed hilar glands and 238 $(28.0 \%)$ had parenchymal silicosis. Thus, the prevalence of gland fibrosis was twice that of parenchymal silicosis. Of the 238 cases with parenchymal silicosis, $209(88 \%)$ had accompanying fibrosed glands-that is, silicosis was more common when fibrosis was present than when it was not. The difference was significant $(Z=11.23 ; p<0.0001)$. The cases were further categorised according to the duration of exposure to mine dust. Forty seven

Table 1 Cases according to presence or absence of parenchymal silicosis and fibrosed hilar glands

\begin{tabular}{llll}
\hline \multicolumn{4}{c}{ Fibrosed glands } \\
\cline { 2 - 4 } Silicosis & Present & Absent & Total \\
\hline Present & 209 & 29 & 238 \\
Absent & 274 & 337 & 611 \\
Total & 483 & 366 & 849 \\
\hline
\end{tabular}

Table 3 Odds ratios for the variables in the logistic regression model

\begin{tabular}{lll}
\hline & Odds ratio & $95 \%$ CI \\
\hline Gland fibrosis & $2 \cdot 56$ & $1 \cdot 05-3 \cdot 18$ \\
Dust exposure (y): & & \\
1 & 1.04 & $1 \cdot 02-1 \cdot 05$ \\
5 & $1 \cdot 20$ & $1 \cdot 18-1 \cdot 21$ \\
10 & 1.43 & $1 \cdot 41-1 \cdot 46$ \\
20 & $2 \cdot 06$ & $2 \cdot 02-2 \cdot 09$ \\
30 & 2.95 & $2 \cdot 91-3 \cdot 00$ \\
40 & $4 \cdot 24$ & $4 \cdot 17-4 \cdot 30$ \\
\hline
\end{tabular}

${ }^{\star} \mathrm{p}<0.0001$.

$(5.5 \%)$ of the 849 cases had no information regarding duration of exposure and were therefore excluded from further analysis. The remaining 802 cases were divided into five year exposure periods (table 2).

The overall distribution of cases with known duration of exposure was:

(1) fibrosed hilar glands and silicosis- $25.9 \%$;

(2) fibrosed hilar glands, no silicosis- $33.2 \%$;

(3) normal hilar glands and silicosis- $3.5 \%$;

(4) normal hilar glands, no silicosis-37.4\%.

The proportion of cases with both hilar gland fibrosis and silicosis, as well as cases with only hilar gland fibrosis, increased with duration of exposure. The proportion of cases with parenchymal silicosis alone remained fairly constant for all exposure periods. The proportion of cases with neither condition decreased with increasing exposure. Logistic regression analysis was undertaken with silicosis as the dependent (outcome) variable and dust exposure and gland fibrosis as independent (predictor) variables (table 3 ). This model assumes that gland fibrosis precedes silicosis, which appears justified on the basis of the data presented in table 2 . The chance of having parenchymal silicosis was 2.56 times greater for those with gland fibrosis compared with those without. Exposure information, unlike hilar gland fibrosis, was a continuous variable. The odds

Table 2 Distribution of cases by exposure period

\begin{tabular}{|c|c|c|c|c|c|c|c|c|c|}
\hline \multirow[b]{4}{*}{ Exposure period (y) } & \multicolumn{9}{|c|}{ Lymph glands } \\
\hline & \multicolumn{4}{|c|}{ Fibrosed } & \multicolumn{4}{|c|}{ Not fibrosed } & \multirow[b]{3}{*}{ Total } \\
\hline & \multicolumn{2}{|c|}{ Silicosis present } & \multicolumn{2}{|c|}{ Silicosis absent } & \multicolumn{2}{|c|}{ Silicosis present } & \multicolumn{2}{|c|}{ Silicosis absent } & \\
\hline & No & $(\%)$ & No & $(\%)$ & No & $(\%)$ & No & $(\%)$ & \\
\hline $\begin{array}{l}0 \cdot 1-5 \cdot 0 \\
5 \cdot 1-10 \cdot 0 \\
10 \cdot 1-15 \cdot 0 \\
15 \cdot 1-20 \cdot 0 \\
20 \cdot 1-25 \cdot 0 \\
25 \cdot 1-30 \cdot 0 \\
30 \cdot 1-35 \cdot 0 \\
35 \cdot 1-40 \cdot 0 \\
\geqslant 40 \cdot 1\end{array}$ & $\begin{array}{r}1 \\
4 \\
11 \\
15 \\
19 \\
29 \\
50 \\
49 \\
30\end{array}$ & $\begin{array}{l}(1 \cdot 3) \\
(5 \cdot 7) \\
(20 \cdot 0) \\
(21 \cdot 4) \\
(27 \cdot 1) \\
(25 \cdot 0) \\
(37 \cdot 3) \\
(39 \cdot 2) \\
(34 \cdot 5)\end{array}$ & $\begin{array}{l}13 \\
13 \\
18 \\
24 \\
19 \\
54 \\
49 \\
44 \\
32\end{array}$ & $\begin{array}{l}(17 \cdot 3) \\
(18 \cdot 6) \\
(32 \cdot 7) \\
(34 \cdot 3) \\
(27 \cdot 1) \\
(46 \cdot 5) \\
(36 \cdot 6) \\
(35 \cdot 2) \\
(36 \cdot 8)\end{array}$ & $\begin{array}{l}0 \\
3 \\
1 \\
2 \\
4 \\
6 \\
4 \\
4 \\
4\end{array}$ & $\begin{array}{l}(0) \\
(4 \cdot 3) \\
(1 \cdot 8) \\
(2 \cdot 9) \\
(5 \cdot 7) \\
(5 \cdot 2) \\
(3 \cdot 0) \\
(3 \cdot 2) \\
(4 \cdot 6)\end{array}$ & $\begin{array}{l}61 \\
50 \\
25 \\
29 \\
28 \\
27 \\
31 \\
28 \\
21\end{array}$ & $\begin{array}{l}(81 \cdot 3) \\
(71 \cdot 4) \\
(45 \cdot 4) \\
(41 \cdot 4) \\
(40 \cdot 6) \\
(23 \cdot 3) \\
(23 \cdot 1) \\
(22 \cdot 4) \\
(24 \cdot 1)\end{array}$ & $\begin{array}{r}75 \\
70 \\
55 \\
70 \\
70 \\
116 \\
134 \\
125 \\
87\end{array}$ \\
\hline Total & 208 & & 266 & & 28 & & 300 & & 802 \\
\hline
\end{tabular}


ratio for developing silicosis in the presence of hilar gland fibrosis increased from 1.04 at one year to $4.2 \overline{4}$ at 40 years of mine dust exposure.

\section{Discussion}

Recent advances in the understanding of the fundamental mechanisms of the mineral dust diseases have substantially increased our knowledge of the pathogenesis of silicosis. ${ }^{5-8}$ In an occupational setting with dust exposure the risk of developing silicosis is related to the intensity and duration of exposure. ${ }^{2}$ Not all workers in the same environment, however, will develop silicosis, indicating that individual susceptibility may play a part. ${ }^{10}$

Attention has been paid to factors affecting particle clearance. Evidence has been presented that cigarette smoking inhibits mucociliary clearance as well as decreasing alveolar clearance. ${ }^{14}$ It has also been suggested that interstitial fibrosis and emphysema reduce particle clearance. ${ }^{1516}$

Although it has long been recognised that one of the clearance pathways for particles deposited on the alveolar wall concerns the translocation of such particles to the regional lymph glands, the effects of pathological changes in the lymph glands on the development of pneumoconiosis are not known.

The present study attempted to explore the biologically plausible possibility that fibrosis of lymph glands might decrease alveolar clearance of quartz thereby increasing accumulated lung burden and hence the likelihood of developing silicosis. The findings tend to support the hypothesis that hilar gland fibrosis predisposes to parenchymal silicosis, or at least precedes it. There were $274(32.3 \%)$ cases of gland fibrosis without silicosis and $337(39 \cdot 7 \%)$ cases with neither gland fibrosis nor silicosis. Silicosis was present in 238 cases $(28.0 \%$ ) (table 1 ). Of these cases, $209(87.8 \%)$ had accompanying gland fibrosis and only $29(12 \cdot 2 \%)$ showed silicosis without gland fibrosis. If silicosis were to precede gland fibrosis the converse relation would be expected.

When cases were further categorised according to the duration of exposure (table 2) the results showed that with an increase in duration of exposure the proportion of people with gland fibrosis only and those with silicosis and gland fibrosis increased, whereas the proportion with silicosis only remained fairly constant. If gland fibrosis did not precede silicosis we would expect a similar increase in the proportion of people with silicosis only as the duration of exposure increased. Thus, the data presented justify the conclusion that gland fibrosis precedes silicosis.

It is recognised that a major limitation of this study is that it is a prevalence cross sectional study in which the presence of gland fibrosis and silicosis were determined simultaneously at necropsy. No means of conducting a cohort study exists, however, to deter- mine the state of the glands in life at the onset of and during exposure to mine dust.

A possible explanation for the occurrence of cases with lung silicosis and no concurrent gland fibrosis may be quantitative or qualitative deficiences in the sampling of hilar glands; if additional glands had been examined, fibrosis might have been discovered.

While acknowledging the limitations of this study, the findings are presented as evidence implicating gland fibrosis as an important factor in the development of silicosis.

We gratefully acknowledge the work of the pathologists of the National Centre for Occupational Health who performed the necropsies. We thank Drs M Steinberg, P Hessel, and M Ross for their many helpful comments.

Requests for reprints to: Librarian, National Centre for Occupational Health, PO Box 32492, Braamfontein, 2017, Republic of South Africa.

1 Brody AR, Roe MW, Evans JN, Davis GS. Deposition and translocation of inhaled silica in rats, quantification of particle distribution, macrophage participation, and function. Lab Invest 1982;47:533-42.

2 Lippman M, Yeates DB, Albert RE. Deposition, retention, and clearance of inhaled particles. $\mathrm{Br} J$ Ind Med 1980;37:337-62.

3 Lippman M, Schlesinger RB. Interspecies comparisons of particle deposition and mucociliary clearance in tracheobronchial airways. $J$ Toxicol Environ Health 1984;13:441-69.

4 Green GM, Jakob GJ, Low RB, Davis GS. State of the art: defense mechanisms of the respiratory membrane. $A m R e v$ Respir Dis 1977;115:479-514.

5 Harmsen AG, Muggenburg BA, Snipes MB, Bice DE. The role of macrophages in particle translocation from lungs to lymphnodes. Science 1985;230:1277-80.

6 Corry D, Kulkarni P, Lipscomb MF. The migration of bronchoalveolar macrophages into hilar lymph nodes. Am J Pathol 1984;115:321-8.

7 Lehnert BE, Caldez VE, Bomalaski SH. Analyses of particles in the lung free cell, trancheobronchial lymphnodal, and pleural space compartments following their deposition in the lung as related to lung clearance mechanisms. Ann Occup Hyg 1988;32:125-40.

8 Ferin J, Feldstein ML. Pulmonary clearance and hilar lymph node content in rats after particle exposure. Environ Res 1978;16:342-52.

9 Heppleston AG. Silicotic fibrogenesis: A concept of pulmonary fibrosis. Ann Occup Hyg 1982;26:449-62.

10 Davis GS. The pathogenesis of silicosis: state of the art. Chest 1986;89:166-9.

11 Bowden DH, Adamson IYR. The role of cell injury and the continuing inflammatory response in the generation of silicotic pulmonary fibrosis. $J$ Pathol 1984;144:149-61.

12 Hessel PA, Goldstein B, Davies JCA, Webster I, Hnizdo E, Laudau S. Pathological findings in mineworkers. I: Description of the PATHAUT database. Am J Ind Med 1987;12: 71-80.

13 SAS Institute Inc. SAS/STAT user's guide. Release 6.03 ed. Cary, NC: SAS Institute Inc, 1988.

14 Bohning DE, Atkins HL, Cohn SH. Long term particle clearance in man: Normal and Impaired. Ann Occup Hyg 1982;26:259-71.

15 Timbrell V. Deposition and retention of fibres in the human lung. Ann Occup Hyg 1982;26:347-69.

$16 \mathrm{Hahn}$ FF, Hobbs $\mathrm{CH}$. The effects of enzyme induced pulmonary emphysema in Syrian hamsters on the deposition and long term retention of inhaled particles. Arch Environ Health 1979;34:203-11.

Accepted 1 October 1990 